Background Cytomegalovirus, which belongs to the herpes viruses group, is the most frequent cause of congenital infection. The fetus may be silently infected in utero, as a result of initial infection or reactivation of a chronic infection in the mother. This may lead to different failures of the child's organs and systems. The presence of antibodies to cytomegalovirus (CMV) in infants, even without clinical signs of infection, often leads to unreasonable medical treatment.

Patients and Methods The parents of the 2-month-old child appealed to the department to verify the correctness of prescribed treatment. A high level of CMV IgG was detected in maternal blood after pregnancy. That's why congenital CMV infection was suspected in 1-month -old infant. His laboratory tests revealed high level of CMV IgG, but CMV DNA wasn't found by PCR in blood, urine, saliva. In spite of this, the baby was diagnosed with congenital CMV infection and was treated with anti-human anticytomegalovirus immunoglobulin (2 doses). However, antibodies titer was at the same level on repeat testing. On physical examination in our department: the condition of the child was satisfactory, cognitive development was normal.

Results Diagnosis of congenital CMV infection isn't correct, according to negative CMV DNA PCR in blood, saliva, urine and lack of clinical manifestation (microcephaly, jaundice, petechial rash, hepatosplenomegaly, hepatitis, pneumonitis, sensorineural hearing loss, etc.). Therefore, further examination and specific immunoglobulin therapy aren't needed, dynamic observation is recommended. The child's condition remains satisfactory at the age of 4 months, there aren't any complaints from his parents.

Conclusion The main diagnostic test of congenital CMV infection is PCR of body fluids, which means that serological research should not be used in routine diagnostics. The detection of CMV IgG in clinically healthy infants isn't a criteria for this diagnose and does not require specific treatment.

\section{DON'T FORGET ABOUT RISK OF PERTUSSIS IN CHILDREN WITH ASTHMA}

Alina Pankova*, Anastasia Lamasova, Ekaterina Dubonosova, Elizaveta Leonova, Vera Kalugina, Anna Alexeeva, Marina Fedoseenko, Leila Namazova-Baranova. Research Institute of Pediatrics and Child Health CCH RAS, Ministry of Science and Higher Education

\subsection{6/archdischild-2021-europaediatrics.31}

Background Pertussis, also known as 'whooping cough,' is a highly contagious respiratory disease, caused by Bordetella pertussis.

Nowadays it primarily affects children too young to have completed the full course of vaccinations, children with chronic lung diseases, teenagers and adults whose immunity has faded. Incidence of pertussis among children 7-14 years old in Russia increased twice in 2019, compared with 2018, possibly due to fading immunity among vaccinated children. However, there is still no pertussis booster vaccination for children over 6 years of age in the Russian National Immunization Schedule.

Case Report A 10-year-old boy, appealed to the department with complaints of paroxysmal nonproductive cough, becoming nocturnal for the last 14 days, without fever. The boy was diagnosed with asthma at age of 7 years, for the last 1,5 years he had asthma remission and hadn't received controller therapy. The boy was vaccinated according to the National
Immunization Schedule of the Russian Federation. At the previous pediatrician examination there wasn't any additional sounds, normal breath during the lungs' auscultation. However, the doctor decided that it was asthma exacerbation caused by a viral disease. Boy was treated with inhaled salmeterol/fluticasone propionate combination without any significant effect: the cough became worse, hacking, nocturnal with "whooping» sounds.

On physical examination difficulty breathing through the nose was detected.

On auscultation, normal breath without any additional sounds were audible over both lungs. The remainder of the physical examination was unremarkable.

Epidemiological anamnesis: The mother of the child had frequent coughing paroxysms for the last 2 weeks.

Results Pertussis was suspected because of the long-term coughing paroxysms, lack of response to bronchial asthma controller therapy, positive epidemiological anamnesis. Bordetella pertussis DNA was detected by PCR in nasopharynx mucus. Also, positive IgM (12.6 IU/mL) and $\operatorname{IgG}(60.8 \mathrm{IU} / \mathrm{mL})$ antibodies to B. pertussis were found in the blood serum. The boy was treated with clarithromycin. Since the beginning of treatment there was significant improvement in health: the cough became rare without whooping.

Conclusion Children with bronchial asthma and lack of response to controller therapy ought to be suspected with whooping cough. Particular attention should be given to schoolchildren and adolescents, especially with chronic lung diseases: vaccination against pertussis at the age of 6 and 1213 years is recommended, possibly due to fading immunity among vaccinated children over 6 years of age.

\section{CLINICAL CASE OF HYPOHYDROTIC ECTODERM DYSPLASIA: SPECIFIC SYMPTOMS FROM THE ENT ORGANS}

S Gubanova*, I Zelenkova, L Namazova-Baranova, A Alekseeva, L Aslamazyan. The Research Institute of Pediatrics and Child Health of the Central Clinical Hospital of the Russian Academy of Sciences of the Ministry of Science and Higher Education of the Russian Federation

\subsection{6/archdischild-2021-europaediatrics.32}

Ectoderm dysplasia is a rare hereditary disease resulting from mutations in genes encoding the development of ectoderm (mainly ectodysplazin-A receptor genes). The most common form is X-linked hypohydrotic ectoderm dysplasia. The prevalence of this form of the disease is estimated from 1.6 to 22 cases per 100,000 newborns. Clinical symptoms are diverse and can be manifested by impaired function of various systems and organs.

Methods Boy X. 1y $6 \mathrm{~m}$ old, vaccinated only against BCG at birth, saw the ENT doctor with complaints of recurring purulent rhinitis with the formation of crusts in the nasal cavity with a fetid odor. Local therapy (decongestants, elimination therapy) had no effect. Examination by an otorhinolaryngologist revealed chronic atrophic rhinitis. Forming nasal septum perforation and epithelial plugs in the external auditory canals were detected, as well as conical teeth.

Local therapy of rhinitis was prescribed with medications containing D-panthenol (for moisturizing the mucous membrane and prevention nasal septum perforation) and an 